\title{
EUS-FNA for Pelvic Lesions: Is This the Final Answer?
}

\author{
Pietro Fusaroli ${ }^{1} \cdot$ Giancarlo Caletti $^{1}$
}

Published online: 12 October 2015

(c) Springer Science+Business Media New York 2015

Soon after the first report in 1992 [1], EUS-FNA became an indispensable tool to obtain tissue diagnosis in the majority of the lesions within its reach [2]. The two main benefits of endoscopic ultrasound (EUS) are its superior accuracy in the detection of lesions $<2-3 \mathrm{~cm}$ in diameter within $4 \mathrm{~cm}$ from the transducer, compared with extracorporeal imaging techniques, and the possibility of tissue acquisition by EUS-guided fine needle aspiration (FNA). EUS-FNA can facilitate diagnosis when tissue is acquired from the primary tumor and is useful for staging when tissue is acquired from suspicious lymph nodes or suspected metastatic lesions. Pancreatic solid and cystic masses and gastrointestinal subepithelial tumors are typical targets of diagnostic EUS-FNA. Non-peritumoral lymph nodes, liver, and adrenal gland lesions are examples of the utility of EUS-FNA for staging. Moreover, EUS-FNA of either a lymph node or a suspected metastasis (e.g., in the liver) may enable simultaneous diagnosis and staging in a patient whose primary tumor cannot be sampled.

EUS-FNA is particularly useful in the lower gastrointestinal tract where few alternatives exist, such as extracorporeal or laparoscopic sampling, for tissue acquisition from pelvic lesions, as opposed to upper gastrointestinal and mediastinal lesions, where percutaneous and surgical techniques are still in use.

The main indications for pelvic EUS include rectal cancer preoperative staging and post-surgical surveillance, the differential diagnosis of subepithelial tumors, the

Pietro Fusaroli

pietro.fusaroli@unibo.it

1 Gastrointestinal Unit, Department of Medical and Surgical Sciences, Imola Hospital, University of Bologna, Via Montericco 4, 40026 Imola, BO, Italy diagnosis and sampling of perirectal solid and cystic masses, and the evaluation of fecal incontinence and perianal disease. Since male and female abnormalities of the genitourinary tract are not usually part of gastroenterologist's armamentarium, they will not be included in the discussion.

In this issue of Digestive Diseases and Sciences, Han et al. [3] draw attention to the accuracy and safety of pelvic EUS-FNA. By means of systematic literature review and meta-analysis during 2000-2014, data were obtained from 10 studies deemed of high quality according to the method of quality assessment of studies and data extraction (QUADAS). Evidently, the investigators scrupulously contacted the corresponding authors of the selected articles in order to extract the individual data for pelvic lesions or to calculate the specific operating characteristics in 7 of the 10 studies. Overall, 246 patients were included in the analysis. The pooled sensitivity and specificity of EUSFNA were $89 \%$ (95\% confidence interval, 83-94\%) and $93 \%$ (95\% confidence interval, 86-97\%), respectively, and the area under the summary receiver operating characteristic (SROC) curve was 0.96. Nonetheless, the authors stressed a potential publication bias indicated by the significant asymmetry of the Deek's funnel plot, as it is often the case in the EUS literature. In other words, since studies with positive results are more likely to be published than the negative ones, the real sensitivity and specificity of EUS-FNA in practice may be lower than the figures reported in this meta-analysis. The overall adverse events rate was $1.7 \%$ with one post-biopsy hemorrhage, which was managed conservatively, and two patients with cystic lesions who developed abscesses after EUS-FNA and required percutaneous drainage.

Just after the end of the consideration period of the metaanalysis, a large American study was published specifically 
addressing the complication rate after lower gastrointestinal tract EUS-FNA [4]. Complete follow-up data were available for 502 patients in which adverse events were graded from 1 to 5 according to the Common Terminology Criteria for Adverse Events (CTCAE). If all incidents after EUS-FNA, including the mildest ones, were counted, the adverse events rate was as high as $20.5 \%$, whereas if only adverse events of serious grades 3-4 (grade 5, corresponding to death, did not occur) were taken into account, the adverse events rate was $5.6 \%$. The latter included pain, bleeding, perforation, and appendicitis. Although not all adverse events in the American study were attributable exclusively to EUS-FNA, since some patients had also undergone other endoscopic procedures in the same setting, including colonoscopy and polypectomy, it may be assumed that the overall complication rate of pelvic EUS-FNA seems somewhat higher than the 2-6\% rate reported for the upper gastrointestinal tract [5, 6]. Antibiotic prophylaxis prior to EUS-FNA was not systematically adopted by the American investigators except for cystic lesions; instead, complete bowel preparation was always obtained. Interestingly, high fever and abscess formation were not encountered in the study by Levy et al.

Although the figures were different between the metaanalysis of Han et al. and the American large single-center study, probably due to the different numbers of patients enrolled and the definitions of adverse events, the safety of EUS-FNA in the lower gastrointestinal tract and pelvis is clearly of primary importance. As pointed out by Han et al., one of the issues which may prove important is related to the use of echoendoscopes with oblique-viewing optics in a tortuous sigmoid colon. Since these echoendoscopes have been specifically designed for the upper gastrointestinal tract, it is often difficult and risky to insert them more proximally than the recto-sigmoid junction. In this respect, the forward-view echoendoscope recently made available by Olympus might prove useful for EUS examination of the lower gastrointestinal tract proximal the rectum [7].

Since the entirety of the studies analyzed by Han et al. was retrospective, in order to elevate the level of evidence of scientific knowledge in this field, prospective and randomized clinical trials are clearly warranted. Nevertheless, surgical histology represented the 'gold standard' for comparison with EUS in the majority of patients, unlike other studies where the less stringent standard of EUS-FNA cytology plus long-term follow-up was used. As a result, since the methodology of the Han meta-analysis was sound, the data depicted in its forest plots are likely to be reliable.

Prior to EUS-FNA, the study subjects underwent a variety of imaging and endoscopic techniques, including colonoscopy, computed tomography, resonance magnetic imaging, and positron emission tomography. In the writers' experience, it is not infrequent to perform EUS-FNA in patients who have undergone all of the above-mentioned techniques without a definitive diagnosis. If employed earlier in the diagnostic algorithm, EUS-FNA may eliminate the need for multiple expensive tests for benign and malignant conditions. In this respect, suspicion of recurrent perirectal malignancy represents one of the best indications for EUS-FNA, which enables diagnostic confirmation impossible to achieve by any other technique. A recent study from Spain reported excellent positive and negative predictive value of EUS-FNA in 58 patients with a suspicion of perirectal recurrence of colorectal cancer [8]. Interestingly, EUS-FNA confirmed the presence of malignancy in $67 \%$, whereas neoplastic recurrence was excluded in $31 \%$, proving its diagnostic worth in this setting.

On a last note, EUS image enhancement techniques such as contrast harmonic and elastography, which have become established in the diagnosis of pancreatic neoplasms [9], may prove useful in EUS of pelvic diseases as well. For instance, since some subepithelial tumors exhibit different patterns using contrast-harmonic EUS, EUSFNA may be used selectively only when malignancy is suspected. The same applies to elastography, which can be used preliminarily to interrogate numerous perirectal lymph nodes in order to target EUS-FNA sampling of the most worrisome lesions. Ideally, contrast-harmonic EUS and EUS elastography may prove useful in the selective use of EUS-FNA for the diagnosis of pelvic diseases, further enhancing its diagnostic accuracy while reducing the adverse events rate.

\section{Compliance with ethical standards}

Conflict of interest The authors declare no conflict of interest.

\section{References}

1. Vilmann P, Jacobsen GK, Henriksen FW, Hancke S. Endoscopic ultrasonography with guided fine needle aspiration biopsy in pancreatic disease. Gastrointest Endosc. 1992;38:172-173.

2. Wani S, Muthusamy VR, Komanduri S. EUS-guided tissue acquisition: an evidence-based approach (with videos). Gastrointest Endosc. 2014;80:939-959.

3. Han C, Lin R, Liu J, Hou X, Ding Z. Endoscopic ultrasonographyguided biopsy for differentiation of benign and malignant pelvic lesions: a systematic review and metaanalysis. Dig Dis Sci. (Epub ahead of print). doi:10.1007/s10620-015-3831-5.

4. Levy MJ, Abu Dayyeh BK, Fujii LL, et al. Prospective evaluation of adverse events following lower gastrointestinal tract EUS FNA. Am J Gastroenterol. 2014;109:676-685.

5. Tarantino I, Fabbri C, Di Mitri R, et al. Complications of endoscopic ultrasound fine needle aspiration on pancreatic cystic lesions: final results from a large prospective multicenter study. Dig Liver Dis. 2014;46:41-44.

6. Rodríguez-D’Jesús A, Fernández-Esparrach G, Marra-Lopez C, et al. Adverse events of EUS-guided fine-needle aspiration of pancreatic cystic and solid lesions using the lexicon proposed in an 
ASGE workshop: a prospective and comparative study. Gastrointest Endosc. 2015. doi:10.1016/j.gie.2015.08.035.

7. Fusaroli P, Ceroni L, Caletti G. Forward-view endoscopic ultrasound: a systematic review of diagnostic and therapeutic applications. Endosc Ultrasound. 2013;2:64-70.

8. Fernández-Esparrach G, Alberghina N, Subtil JC, et al. Endoscopic ultrasound-guided fine needle aspiration is highly accurate for the diagnosis of perirectal recurrence of colorectal cancer. Dis Colon Rectum. 2015;58:469-473.

9. Gincul R, Palazzo M, Pujol B, et al. Contrast-harmonic endoscopic ultrasound for the diagnosis of pancreatic adenocarcinoma: a prospective multicenter trial. Endoscopy. 2014;46:373-379. 